\title{
Characterization for Rectifiable and Nonrectifiable Attractivity of Nonautonomous Systems of Linear Differential Equations
}

\author{
Yūki Naito ${ }^{1}$ and Mervan Pašićc \\ ${ }^{1}$ Ehime University, Matsuyama 790-8577, Japan \\ ${ }^{2}$ Department of Mathematics, University of Zagreb, FER, 10000 Zagreb, Croatia \\ Correspondence should be addressed to Yūki Naito; ynaito@ehime-u.ac.jp
}

Received 2 April 2013; Accepted 21 May 2013

Academic Editor: Norio Yoshida

Copyright (C) 2013 Y. Naito and M. Pašić. This is an open access article distributed under the Creative Commons Attribution License, which permits unrestricted use, distribution, and reproduction in any medium, provided the original work is properly cited.

We study a new kind of asymptotic behaviour near $t=0$ for the nonautonomous system of two linear differential equations: $\mathbf{x}^{\prime}(t)=A(t) \mathbf{x}(t), t \in\left(0, t_{0}\right]$, where the matrix-valued function $A=A(t)$ has a kind of singularity at $t=0$. It is called rectifiable (resp., nonrectifiable) attractivity of the zero solution, which means that $\|\mathbf{x}(t)\|_{2} \rightarrow 0$ as $t \rightarrow 0$ and the length of the solution curve of $\mathbf{x}$ is finite (resp., infinite) for every $\mathbf{x} \neq \mathbf{0}$. It is characterized in terms of certain asymptotic behaviour of the eigenvalues of $A(t)$ near $t=0$. Consequently, the main results are applied to a system of two linear differential equations with polynomial coefficients which are singular at $t=0$.

\section{Introduction}

We are concerned with the two-dimensional nonautonomous linear differential systems:

$$
\frac{d \mathbf{x}}{d t}=A(t) \mathbf{x} \quad \text { for } t \in\left(0, t_{0}\right], \quad \mathbf{x}\left(t_{0}\right)=\mathbf{x}_{0} \in \mathbb{R}^{2},
$$

where $t_{0}>0$, and $A=A(t), A:\left(0, t_{0}\right] \rightarrow \mathscr{M}_{2}$ is a matrixvalued function such that $A \in C^{1}\left(\left(0, t_{0}\right] ; \mathscr{M}_{2}\right)$, where $\mathscr{M}_{2}$ denotes the space of all $2 \times 2$ real matrices. A solution of linear system (1) is a function $\mathbf{x}=\mathbf{x}(t), \mathbf{x}:\left(0, t_{0}\right] \rightarrow \mathbb{R}^{2}$ such that $\mathbf{x} \in C^{1}\left(\left(0, t_{0}\right], \mathbb{R}^{2}\right)$.

In appendix of the paper, we show, there exists a unique solution $\mathbf{x}$ of linear system (1). By the uniqueness of solution $\mathbf{x}$ of $(1)$, it is clear that if $\mathbf{x}_{0}=(0,0)$, then $\mathbf{x}(t)=(0,0)$ for all $t \in\left(0, t_{0}\right]$. It is called the zero solution of linear system (1). The zero solution of linear system (1) is said to be attractive as $t \rightarrow 0$ if for every $\mathbf{x}_{0} \in \mathbb{R}^{2}$ the corresponding solution $\mathbf{x}$ of (1) satisfies $\|\mathbf{x}(t)\|_{2} \rightarrow 0$ as $t \rightarrow 0$, where $\|\cdot\|_{2}$ denotes the standard Euclidean norm on $\mathbb{R}^{2}$. Since we are dealing with the attractive zero solution of (1), from $\mathbf{x} \in C^{1}\left(\left(0, t_{0}\right], \mathbb{R}^{2}\right)$ we conclude that $\mathbf{x} \in C\left(\left[0, t_{0}\right], \mathbb{R}^{2}\right) \cap C^{1}\left(\left(0, t_{0}\right], \mathbb{R}^{2}\right)$. For a continuous function $\mathbf{x}:\left[0, t_{0}\right] \rightarrow \mathbb{R}^{2}$, let $\Gamma_{\mathbf{x}}$ denote a curve in $\mathbb{R}^{2}$ corresponding to $\mathbf{x}$ determined by

$$
\Gamma_{\mathbf{x}}=\mathbf{x}\left(\left[0, t_{0}\right]\right)=\left\{(x, y) \in \mathbb{R}^{2}: x=x(t), y=y(t)\right\},
$$

where $(x(t), y(t)) \equiv \mathbf{x}(t)$. It is often said that $\Gamma_{\mathbf{x}}$ is parametrized by $\mathbf{x}$.

Let $\mathbf{x} \in C\left(\left[0, t_{0}\right] ; \mathbb{R}^{2}\right)$. The curve $\Gamma_{\mathbf{x}} \subseteq \mathbb{R}^{2}$ is said to be a Jordan curve if $\mathbf{x}$ is injective (one-to-one) on $\left[0, t_{0}\right]$. Hence, $\Gamma_{\mathbf{x}}$ is nonself-intersecting; it has two ends and it is a compact connected set in $\mathbb{R}^{2}$. In appendix of the paper, see Theorem A.3; we give some simple sufficient conditions on the matrix-valued function $A=A(t)$ such that the solution curve $\Gamma_{\mathbf{x}}$ of every nontrivial solution of (1) is a Jordan curve in $\mathbb{R}^{2}$. The assumption that $\Gamma_{\mathbf{x}}$ is a Jordan curve is important for the process of measuring the length of $\Gamma_{\mathbf{x}}$ (the rectification of $\Gamma_{x}$ ) as follows. If $\Gamma_{x}$ is a Jordan curve, then the length of $\Gamma_{x}$ is denoted by length $\left(\Gamma_{\mathbf{x}}\right)$ and it is defined as usual by (see [1-3])

$$
\begin{gathered}
\text { length }\left(\Gamma_{\mathbf{x}}\right)=\sup \left\{\sum_{k=1}^{m}\left\|\mathbf{x}\left(t_{k}\right)-\mathbf{x}\left(t_{k-1}\right)\right\|_{2}: m \in \mathbb{N},\right. \\
\left.0=t_{m}<\cdots<t_{1}<t_{0}\right\},
\end{gathered}
$$

where the supremum is taken over all finite dissections $0=$ $t_{m}<\cdots<t_{1}<t_{0}$ of the interval $\left[0, t_{0}\right]$. 
Definition 1. The zero solution of linear system (1) is said to be rectifiable (resp., nonrectifiable) attractive as $t \rightarrow 0$; if for every $\mathbf{x}_{0} \in \mathbb{R}^{2} \backslash\{\mathbf{0}\}$ such that the $\Gamma_{\mathbf{x}}$ of corresponding solution $\mathbf{x}$ of (1) is a Jordan curve in $\mathbb{R}^{2}$, one has $\|\mathbf{x}(t)\|_{2} \rightarrow 0$ as $t \rightarrow 0$ and length $\left(\Gamma_{\mathbf{x}}\right)<\infty$ (resp., length $\left(\Gamma_{\mathbf{x}}\right)=\infty$ ).

Let $\lambda(t)=\operatorname{Re} \lambda(t) \pm i \operatorname{Im} \lambda(t), t \in\left(0, t_{0}\right]$, be two complex conjugate eigenvalues of $A(t)$. The main purpose of the paper is to characterize attractivity, rectifiable and nonrectifiable attractivity, as $t \rightarrow 0$ of the zero solution of system (1) in the terms of regular and singular asymptotic behaviour of $A(t)$ near $t=0$; see Theorems 2 and 3. It is illustrated on a simple two-dimensional linear differential systems with the polynomial coefficients; see Theorem 4. The key point of proofs of the main results is an asymptotic solution formula for system (1) obtained by its asymptotic integration near $t=0$; see Section 3. The proofs of the main results are given in Sections 4, 5, and 6. Such a kind of topics has been considered for the first time in [4] but only in the case of the so-called integrable systems (systems that allow all solutions in explicit forms), where the asymptotic integration near $t=0$ is not required. About the asymptotic integration near $t=\infty$ of differential equations and systems, we refer reader to [5-12]. On several asymptotic properties of twodimensional differential systems near $t=\infty$, let us see, for instance, [13-18]. Recently interest for studying differential equations and systems on compact (finite time) intervals is rapidly increasing because of its application in atmosphere, fluid, and ocean dynamics and in biological science; see, for instance, [19-21] and references therein.

\section{Main Results and Consequences}

Let for each $t \in\left(0, t_{0}\right]$ the matrix elements of $A(t)$ be denoted by $\left(a_{i, j}(t)\right)_{i, j \leq 2}$. In the paper, we suppose the following kind of singular asymptotic behaviour of $A(t)$ near $t=0$ :

$$
\begin{gathered}
\lim _{\varepsilon \rightarrow 0} \int_{\varepsilon}^{t_{0}}\left|\frac{d}{d t}\left(t^{2} a_{i, j}(t)\right)\right| d t<\infty, \quad i, j \in\{1,2\}, \\
\lim _{t \rightarrow 0}\left[t^{2} A(t)\right]=A_{0},
\end{gathered}
$$

where $A_{0}$ is a constant matrix having two complex conjugate eigenvalues.

Some essential consequences of (4) are presented in Section 3. Since the eigenvalues of $A(t)$ are continuous on $t \in\left(0, t_{0}\right]$, by (4) we may assume that $A(t)$ has two complex conjugate eigenvalues $\lambda(t)=\operatorname{Re} \lambda(t) \pm i \operatorname{Im} \lambda(t)$ for $t \in\left(0, t_{0}\right]$.

The first two main results of the paper are the following theorems.

Theorem 2. Let (4) be satisfied, and let $\lambda(t)=\operatorname{Re} \lambda(t) \pm$ $i \operatorname{Im} \lambda(t), t \in\left(0, t_{0}\right]$, be two complex conjugate eigenvalues of $A(t)$. Assume that $\operatorname{Re} \lambda(t) \geq 0$ near $t=0$. Then the zero solution of linear system (1) is attractive as $t \rightarrow 0$ if and only if

$$
\lim _{t \rightarrow 0} \int_{t}^{t_{0}} \operatorname{Re} \lambda(s) d s=\infty .
$$

Theorem 3. Let (4) be satisfied, and let $\lambda(t)=\operatorname{Re} \lambda(t) \pm$ $i \operatorname{Im} \lambda(t), t \in\left(0, t_{0}\right]$, be two complex conjugate eigenvalues of $A(t)$. Assume that $\operatorname{Re} \lambda(t) \geq 0$ near $t=0$, and that (5) holds. Then the zero solution of linear system (1) is rectifiable attractive as $t \rightarrow 0$ if and only if

$$
\operatorname{Im} \lambda(t) e^{-\int_{t}^{t_{0}} \operatorname{Re} \lambda(s) d s} \in L^{1}\left(0, t_{0}\right) .
$$

Previous theorem allows us to study the rectifiable and nonrectifiable attractivity for the following model system with polynomial singular coefficients near $t=0$ :

$$
\begin{gathered}
x^{\prime}(t)=e(t) t^{-p} x(t)+f(t) t^{-2} y(t), \\
y^{\prime}(t)=-g(t) t^{-2} x(t)+h(t) t^{-q} y(t), \quad t \in\left(0, t_{0}\right],
\end{gathered}
$$

where the parameters $p, q \in \mathbb{R}$ and coefficients $e, f, g, h \in$ $C\left(\left[0, t_{0}\right]\right) \cap C^{1}\left(\left(0, t_{0}\right]\right)$ satisfy, respectively,

$$
\begin{gathered}
1 \leq \max \{p, q\}<2, \\
f(0) g(0)>0, \quad e(t)>0, \quad h(t)>0, \quad t \in\left[0, t_{0}\right], \\
\left\{\begin{array}{c}
\int_{0}^{t_{0}}\left|f^{\prime}(t)\right| d t<\infty, \quad \int_{0}^{t_{0}}\left|g^{\prime}(t)\right| d t<\infty, \\
\int_{0}^{t_{0}}\left|\frac{d}{d t}\left(t^{2-p} e(t)\right)\right| d t<\infty, \\
\int_{0}^{t_{0}}\left|\frac{d}{d t}\left(t^{2-q} h(t)\right)\right| d t<\infty .
\end{array}\right.
\end{gathered}
$$

According to (8), (9), and (10), we will show that the model system (7) satisfies all assumptions of Theorem 3. Hence, we are able to derive and prove the following conditions for the rectifiable and nonrectifiable attractivity of the zero solution of model system (7).

Theorem 4. Let (8), (9), and (10) be fulfilled.

(i) If $\max \{p, q\}>1$, then the zero solution of (7) is rectifiable attractive as $t \rightarrow 0$.

(ii) If $\max \{p, q\}=1$, then the zero solution of (7) is rectifiable attractive as $t \rightarrow 0$ provided that one of the following conditions is satisfied:

$$
\begin{array}{ll}
p=1, \quad q<1, \quad e(t)>2 ; \\
p<1, \quad q=1, \quad h(t)>2 ; \\
p=1, \quad q=1, \quad e(t)+h(t)>2 .
\end{array}
$$

(iii) If $\max \{p, q\}=1$, then the zero solution of (7) is nonrectifiable attractive as $t \rightarrow 0$ provided that one of the following conditions is satisfied:

$$
\begin{array}{lll}
p=1, & q<1, & e(t) \leq 2 ; \\
p<1, & q=1, \quad h(t) \leq 2 \\
p=1, & q=1, \quad e(t)+h(t) \leq 2 .
\end{array}
$$


As a consequence of previous theorem, we realize that the rectifiable and nonrectifiable attractivity of the zero solution of model system (7) depends on the order of growth of singular behaviour of coefficients near $t=0$ determined by parameters $p$ and $q$. Let us remark that if, besides (8), (9), and (10), it is supposed that $e(t) \equiv h(t), f(t) \equiv g(t)$, and $p=q$, then system (7) is integrable, that is, all solutions of system (7) are explicitly determined, see appendix of the paper. In such a particular case of (7), Theorem 4 can be explicitly verified.

Remark 5. We suggest reader to consider the rectifiable and nonrectifiable attractivity of the zero solution of system (7) in the case of $\max \{p, q\}=2$. It is required to assume that $4 f(0) g(0)-e^{2}(0)>0$ if $p=2>q$ as well as $4 f(0) g(0)-$ $h^{2}(0)>0$ if $q=2>p$ and $4 f(0) g(0)-(e(0)-h(0))^{2}>0$ if $p=q=2$.

The most difficult point of this paper is to derive and prove the nonrectifiable attractivity of the zero solution of systems (1) and (7); see Sections 3 and 4. On the (non)rectifiability of the solution curve of scalar second-order differential equations, we refer reader to [22-27].

\section{Some Asymptotic Properties Near $t=0$}

As a consequence of the main assumption (4), we derive in this section some important asymptotic properties of $A(t)$ as well as of its eigenvalues and solutions of system (1), which will be used in the next sections in the proof of the main results. Since the eigenvalues of $A(t)$ are continuous on $\left(0, t_{0}\right]$, by (4) we may assume that $A(t)$ has two complex conjugate eigenvalues $\lambda(t)=\operatorname{Re} \lambda(t) \pm i \operatorname{Im} \lambda(t), t \in\left(0, t_{0}\right]$.

Lemma 6. Let (4) be satisfied. Then the eigenvalues of $A(t)$ satisfy

$$
\begin{gathered}
\|A(t)\| \sim|\operatorname{Im} \lambda(t)| \sim t^{-2} \quad \text { as } t \longrightarrow 0, \\
\operatorname{Im} \lambda(t) \neq 0 \quad \text { near } t=0,
\end{gathered}
$$

where $f(t) \sim g(t)$ means that $f(t) / g(t) \rightarrow 1$ as $t \rightarrow 0$.

Proof. Let $a_{i, j}=a_{i, j}(t), a_{i, j} \in C^{1}\left(\left(0, t_{0}\right]\right)$, be matrix elements of $A(t)$. Also, let $a_{i, j}^{0}$ and $\lambda_{0}=\operatorname{Re} \lambda_{0} \pm i \operatorname{Im} \lambda_{0}$ be matrix elements and eigenvalues of $A_{0}$, respectively, where $A_{0}$ appears in (4). Rewriting (4) in terms of matrix elements and eigenvalues, we get

$$
\lim _{t \rightarrow 0}\left[t^{2} a_{i, j}(t)\right]=a_{i, j}^{0}, \quad \operatorname{Im} \lambda_{0} \neq 0 .
$$

Hence, we can conclude the following:

$$
\begin{aligned}
\lim _{t \rightarrow 0} & {\left[t^{2}|\operatorname{Im} \lambda(t)|\right] } \\
\quad= & \frac{1}{2} \lim _{t \rightarrow 0}\left[t^{2} \sqrt{-\left(a_{1,1}(t)-a_{2,2}(t)\right)^{2}-4 a_{1,2}(t) a_{2,1}(t)}\right]
\end{aligned}
$$

$$
\begin{aligned}
& =\frac{1}{2} \lim _{t \rightarrow 0} \sqrt{-\left(t^{2} a_{1,1}(t)-t^{2} a_{2,2}(t)\right)^{2}-4 t^{2} a_{1,2}(t) t^{2} a_{2,1}(t)} \\
& =\frac{1}{2} \sqrt{-\left(a_{1,1}^{0}-a_{2,2}^{0}\right)^{2}-4 a_{1,2}^{0} a_{2,1}^{0}}=\left|\operatorname{Im} \lambda_{0}\right| \neq 0 .
\end{aligned}
$$

It proves that $|\operatorname{Im} \lambda(t)| \sim t^{-2}$ as $t \rightarrow 0$. Hence (18) and the second claim in (17) hold. In order to complete (17), it rests to show that $\|A(t)\| \sim t^{-2}$ as $t \rightarrow 0$. In fact, from the assumption $\operatorname{Im} \lambda_{0} \neq 0$, we observe that for at least one matrix element $a_{i, j}^{0}$ we have $a_{i, j}^{0} \neq 0$ and, therefore, $\left\|A_{0}\right\| \neq 0$. Moreover, since every matrix norm is a continuous function, from (4) we especially obtain $\lim _{t \rightarrow 0}\left[t^{2}\|A(t)\|\right]=\left\|A_{0}\right\| \neq 0$. It completes the proof of (17).

As the main consequence of (4) we have the following asymptotic formula near $t=0$ for all solutions $\mathbf{x}$ of linear system (1):

$$
\begin{aligned}
\mathbf{x}(t)=e^{-\int_{t}^{t_{0}} \operatorname{Re} \lambda(s) d s}\left[\mathbf{h}_{1}(t) \cos \left(\int_{t}^{t_{0}} \operatorname{Im} \lambda(s) d s\right)\right. \\
\left.+\mathbf{h}_{2}(t) \sin \left(\int_{t}^{t_{0}} \operatorname{Im} \lambda(s) d s\right)\right],
\end{aligned}
$$

where $\mathbf{h}_{1}=\mathbf{h}_{1}(t), \mathbf{h}_{2}=\mathbf{h}_{2}(t), \mathbf{h}_{i}:\left(0, t_{0}\right] \rightarrow \mathbb{R}^{2}$ and there are $\mathbf{c}_{1}, \mathbf{c}_{2} \in \mathbb{R}^{2}$ such that

$$
\lim _{t \rightarrow 0} \mathbf{h}_{i}(t)=\mathbf{c}_{i} \neq \mathbf{0}, \quad i \in\{1,2\}
$$

It is the subject of the next lemma.

Lemma 7. Let $\lambda(t)=\operatorname{Re} \lambda(t) \pm i \operatorname{Im} \lambda(t)$ be two eigenvalues of $A(t)$. If $A(t)$ satisfies (4), then for every solution $\mathbf{x}$ of linear system (1) with $\mathbf{x}_{0} \neq\{\mathbf{0}\}$ there exist two functions $\mathbf{h}_{i}(t)$ and two constants $\mathbf{c}_{1}, \mathbf{c}_{2} \in \mathbb{R}^{2}$ such that (21) and (22) are fulfilled.

Proof. Let $A(t)$ be a matrix satisfying all assumptions of this lemma. Let $B(\tau)=\left(b_{i, j}(\tau)_{i, j \leq 2}\right)$ be a new matrix depending on a new variable $\tau:=1 / t$ defined by

$$
B(\tau)=-\tau^{-2} A\left(\tau^{-1}\right) \quad \forall \tau \geq \tau_{0}:=\frac{1}{t_{0}}
$$

Then $b_{i, j} \in C^{1}\left(\left[\tau_{0}, \infty\right)\right)$. Let $A_{0}$ be a constant matrix determined in (4). If $B_{0}$ is a new matrix defined by $B_{0}=-A_{0}$, then from (4), (23), and $t=1 / \tau$, we conclude

$$
\begin{gathered}
\lim _{M \rightarrow \infty} \int_{\tau_{0}}^{M}\left|\frac{d}{d \tau} b_{i, j}(\tau)\right| d \tau<\infty, \quad i, j \in\{1,2\}, \\
\lim _{\tau \rightarrow \infty} B(\tau)=B_{0},
\end{gathered}
$$

where $B_{0}$ is a constant matrix having two complex conjugate eigenvalues. 
Also, the eigenvalues $\mu_{1}(\tau), \mu_{2}(\tau)$ of $B(\tau)$ and $\lambda_{1}(t)=$ $\operatorname{Re} \lambda(t)+i \operatorname{Im} \lambda(t), \lambda_{2}(t)=\operatorname{Re} \lambda(t)-i \operatorname{Im} \lambda(t)$ of $A(t)$ are obviously related by the equality

$$
\begin{aligned}
\mu_{i}(\tau) & =-\tau^{-2} \lambda_{i}\left(\tau^{-1}\right) \\
& =-\tau^{-2}\left[\operatorname{Re}\left(\lambda\left(\tau^{-1}\right)\right) \pm i \operatorname{Im}\left(\lambda\left(\tau^{-1}\right)\right)\right], \quad i=1,2
\end{aligned}
$$

and hence,

$$
\operatorname{Re}\left(\mu_{1}(\tau)-\mu_{2}(\tau)\right)=0 \quad \forall \tau \geq \tau_{0} .
$$

Putting $t=1 / \tau$ into system (1) and denoting by

$$
\mathbf{w}(\tau):=\mathbf{x}\left(\frac{1}{\tau}\right) \quad \text { for } \tau \geq \tau_{0},
$$

we obtain the equivalent system of (1)

$$
\mathbf{w}^{\prime}(\tau)=B(\tau) \mathbf{w}(\tau) \quad \text { for } \tau \geq \tau_{0},
$$

where matrix $B(\tau)$ is defined in (23). For system (28), we will use the next generalized Matell's theorem appearing as Theorem 11 in Coppel's book [5, Chapter 4] and Theorem 6.5 in Kiguradze's monograph [7] (see also [12, Theorem 7] and [8]).

Theorem 8 (see [7]). Let $\mu_{1}=\mu_{1}(\tau)$ and $\mu_{2}=\mu_{2}(\tau)$, $\mu_{1}, \mu_{2}:\left[\tau_{0}, \infty\right) \rightarrow \mathbb{C}$ be two eigenvalues of an arbitrary $2 \times 2$ matrix $B(\tau), \tau \in\left[\tau_{0}, \infty\right)$, with the matrix elements $b_{i, j}(\tau)$ which are absolutely continuous functions on every compact set $I \subset\left[\tau_{0}, \infty\right)$ and

$$
\int_{\tau_{0}}^{\infty}\left|\frac{d}{d \tau} b_{i, j}(\tau)\right| d \tau<\infty, \quad i, j \in\{1,2\} .
$$

Let $c>0$ be a constant such that

$$
\begin{aligned}
& \int_{\sigma_{*}}^{\tau_{*}} \operatorname{Re}\left(\mu_{1}(\tau)-\mu_{2}(\tau)\right) d \tau \leq c, \quad \tau_{0} \leq \sigma_{*}<\tau_{*}, \text { or } \\
& \int_{\tau_{0}}^{\infty} \operatorname{Re}\left(\mu_{1}(\tau)-\mu_{2}(\tau)\right) \mathrm{d} \tau=\infty, \\
& \int_{\sigma_{*}}^{\tau_{*}} \operatorname{Re}\left(\mu_{1}(\tau)-\mu_{2}(\tau)\right) d \tau \geq-c, \quad \tau_{0} \leq \sigma_{*}<\tau_{*} .
\end{aligned}
$$

Let $B(\tau) \rightarrow B_{0}$ as $\tau \rightarrow \infty$, where $B_{0}$ is a constant matrix having two different eigenvalues. Then linear differential system (28) has a fundamental system of solutions $\mathbf{w}_{1}(\tau) \in \mathbb{C}^{2}$ and $\mathbf{w}_{2}(\tau) \in \mathbb{C}^{2}$ such that

$$
\begin{aligned}
& \lim _{\tau \rightarrow \infty}\left[\mathbf{w}_{1}(\tau) e^{-\int_{\tau_{0}}^{\tau} \mu_{1}(\sigma) d \sigma}\right]=\mathbf{b}_{1}, \\
& \lim _{\tau \rightarrow \infty}\left[\mathbf{w}_{2}(\tau) e^{-\int_{\tau_{0}}^{\tau} \mu_{2}(\sigma) d \sigma}\right]=\mathbf{b}_{2},
\end{aligned}
$$

where $\mathbf{b}_{1}, \mathbf{b}_{2} \in \mathbb{C}^{2}$ are two eigenvectors of constant matrix $B_{0}$.

Now, with the help of (24) and (26), it is easy to check that the matrix $B(\tau)$ defined in (23) and its eigenvalues $\mu_{1}(\tau)$ and $\mu_{2}(\tau)$ defined in (25) satisfy all required assumptions of Theorem 8 . Hence, by Theorem 8 , we observe that linear system (1) has a fundamental system of solutions $\mathbf{x}_{1}(t)$ and $\mathbf{x}_{2}(t)$ satisfying

$$
\begin{aligned}
& \lim _{t \rightarrow 0}\left[\mathbf{x}_{1}(t) e^{\int_{t}^{t_{0}} \lambda_{1}(s) d s}\right]=\mathbf{b}_{1}, \\
& \lim _{t \rightarrow 0}\left[\mathbf{x}_{2}(t) e^{\int_{t}^{t_{0}} \lambda_{2}(s) d s}\right]=\mathbf{b}_{2} .
\end{aligned}
$$

Indeed, from (31) and using $\tau=1 / t, \tau_{0}=1 / t_{0},(25),(27)$, and $\sigma=1 / s$, we obtain

$$
\begin{aligned}
\mathbf{b}_{1} & =\lim _{\tau \rightarrow \infty}\left[\mathbf{w}_{1}(\tau) e^{-\int_{\tau_{0}}^{\tau} \mu_{1}(\sigma) d \sigma}\right] \\
& =\lim _{t \rightarrow 0}\left[\mathbf{w}_{1}\left(\frac{1}{t}\right) e^{-\int_{1 / t_{0}}^{1 / t} \mu_{1}(\sigma) d \sigma}\right] \\
& =\lim _{t \rightarrow 0}\left[\mathbf{w}_{1}\left(\frac{1}{t}\right) e^{\int_{1 / t_{0}}^{1 / t} \sigma^{-2} \lambda_{1}\left(\sigma^{-1}\right) d \sigma}\right] \\
& =\lim _{t \rightarrow 0}\left[\mathbf{x}_{1}(t) e^{\int_{t}^{t_{0}} \lambda_{1}(s) d s}\right] .
\end{aligned}
$$

Analogously, the second equality in (32) can be proved. Now, from (32) we particularly obtain

$$
\begin{aligned}
& \lim _{t \rightarrow 0}\left[\mathbf{x}_{1}(t) e^{\int_{t}^{t_{0}} \lambda_{1}(s) d s}-\mathbf{b}_{1}\right]=\mathbf{0}, \\
& \lim _{t \rightarrow 0}\left[\mathbf{x}_{2}(t) e^{\int_{t}^{t_{0}} \lambda_{2}(s) d s}-\mathbf{b}_{2}\right]=\mathbf{0} .
\end{aligned}
$$

Denoting

$$
\begin{aligned}
& \mathbf{g}_{1}(t)=\mathbf{x}_{1}(t) e^{\int_{t}^{t_{0}} \lambda_{1}(s) d s}-\mathbf{b}_{1}, \\
& \mathbf{g}_{2}(t)=\mathbf{x}_{2}(t) e^{\int_{t}^{t_{0}} \lambda_{2}(s) d s}-\mathbf{b}_{2},
\end{aligned}
$$

from (34), we get $\lim _{t \rightarrow 0} \mathbf{g}_{i}(t)=\mathbf{0}, i \in\{1,2\}$ and near $t=0$,

$$
\begin{aligned}
& \mathbf{x}_{1}(t)=\left(\mathbf{b}_{1}+\mathbf{g}_{1}(t)\right) e^{-\int_{t}^{t_{0}} \lambda_{1}(s) d s}, \\
& \mathbf{x}_{2}(t)=\left(\mathbf{b}_{2}+\mathbf{g}_{2}(t)\right) e^{-\int_{t}^{t_{0}} \lambda_{2}(s) d s} .
\end{aligned}
$$

Since $\lambda_{1}(t)=\operatorname{Re} \lambda(t)+i \operatorname{Im} \lambda(t), \lambda_{2}(t)=\operatorname{Re} \lambda(t)-i \operatorname{Im} \lambda(t)$, $\mathbf{b}_{i} \in \mathbb{C}^{2}$ and $\mathbf{g}_{i}:\left(0, t_{0}\right] \rightarrow \mathbb{C}^{2}$, previous statements verify the desired asymptotic formula (21). The statement (22) holds because $\mathbf{b}_{1}$ and $\mathbf{b}_{2}$ are two eigenvectors and hence, $\mathbf{b}_{i} \neq \mathbf{0}$.

Now, according to the asymptotic formula (21), we are able to prove Theorem 2 .

Proof of Theorem 2. Because of (4) and Lemmas 6 and 7, we can use (17) and (18) and the asymptotic solution's formula (21)-(22).

At first, we suppose that (5) holds. Taking the norm on both sides in (21) and using (22), we obtain the upper estimate

$$
\|\mathbf{x}(t)\|_{2} \leq c e^{-\int_{t}^{t_{0}} \operatorname{Re} \lambda(s) d s} \text { near } t=0
$$


with some constant $c>0$. Now putting the limit as $t \rightarrow 0$ on both sides in (37), and using (5), we get $\|x(t)\|_{2} \rightarrow 0$ as $t \rightarrow 0$. Thus, it is shown that the zero solution of system (1) is attractive as $t \rightarrow 0$ provided that (5) holds.

The proof of the reverse claim is slightly complicated. Suppose that

$$
\|\mathbf{x}(t)\|_{2} \longrightarrow 0 \quad \text { as } t \longrightarrow 0 .
$$

Next, from (17), and (18), we especially obtain

$$
\lim _{t \rightarrow 0}\left|\int_{t}^{t_{0}} \operatorname{Im} \lambda(s) d s\right|=\infty .
$$

Remarking that in (21) and (22), we use the following notations:

$$
\begin{gathered}
\mathbf{x}(t)=(x(t), y(t)), \quad \mathbf{h}_{i}(t)=\left(h_{i, 1}(t), h_{i, 2}(t)\right), \\
\mathbf{c}_{i}=\left(c_{i, 1}, c_{i, 2}\right), \quad i \in\{1,2\} .
\end{gathered}
$$

By (22), at least one of $c_{i, 1}$ and $c_{i, 2}$ is nonzero. So, for instance, $c_{1,1} \neq 0$, which together by (22) gives

$$
\lim _{t \rightarrow 0}\left|h_{1,1}(t)\right|=\left|c_{1,1}\right|>0 .
$$

Now, from (21) and (40), we derive the following inequality:

$$
\begin{aligned}
\|\mathbf{x}(t)\| \geq\|\mathbf{x}(t)\| & \\
=e^{-\int_{t}^{t_{0}} \operatorname{Re} \lambda(s) d s} \mid & h_{1,1}(t) \cos \left(\int_{t}^{t_{0}} \operatorname{Im} \lambda(s) d s\right) \\
& +h_{1,2}(t) \sin \left(\int_{t}^{t_{0}} \operatorname{Im} \lambda(s) d s\right) \mid .
\end{aligned}
$$

Next, because of (18) and (39), there is a sequence $t_{n} \in\left(0, t_{0}\right]$, $t_{n} \rightarrow 0$ as $n \rightarrow \infty$, such that

$$
\left|\int_{t_{n}}^{t_{0}} \operatorname{Im} \lambda(s) d s\right|=n \pi, \quad n \in \mathbb{N} .
$$

Now, putting $t=t_{n}$ into (42) and using (41), (43), and $\sin ( \pm n \pi)=0$, we get the inequality

$$
\left\|\mathbf{x}\left(t_{n}\right)\right\|_{2} \geq \frac{\left|c_{1,1}\right|}{2} e^{-\int_{t_{n}}^{t_{0}} \operatorname{Re} \lambda(s) d s \quad \forall \text { large } n \in \mathbb{N} .}
$$

Taking the limit on both sides in previous inequality, with the help of (38), we conclude that

$$
\lim _{n \rightarrow \infty} e^{-\int_{t_{n}}^{t_{0}} \operatorname{Re} \lambda(s) d s}=0
$$

that is,

$$
\lim _{n \rightarrow \infty} \int_{t_{n}}^{t_{0}} \operatorname{Re} \lambda(s) d s=\infty .
$$

Since $\operatorname{Re} \lambda(t) \geq 0$ near $t=0$, the function $\int_{t}^{t_{0}} \operatorname{Re} \lambda(s) d s$ is continuous (since matrix elements of $A(t)$ are continuous functions on $\left.\left(0, t_{0}\right]\right)$ and monotone. Hence, statement (46) proves (5). Thus, the statement (5) holds provided that the zero solution of system (1) is attractive.

\section{Rectifiable Attractivity of the Zero Solution}

Unlike the nonrectifiable attractivity which will be studied in the Section 5, the rectifiable attractivity of the zero solution is proved without any essential difficulties. It is because we can use here the following known result.

Lemma 9. Let $\Gamma_{\mathbf{x}}$ be a Jordan curve in $\mathbb{R}^{2}$. Then one has length $\left(\Gamma_{\mathbf{x}}\right)<\infty$ if and only if $\mathbf{x}^{\prime} \in L^{1}\left(\left(0, t_{0}\right), \mathbb{R}^{2}\right)$.

For the proof of previous lemma, we suggest reader to consult the appropriate scalar case shown in [28].

The following theorem derives some properties of $A(t)$ and its eigenvalues $\lambda(t)$ which ensure that the zero solution of system (1) is rectifiable attractive.

Theorem 10. Let $\lambda(t)=\operatorname{Re} \lambda(t) \pm i \operatorname{Im} \lambda(t)$ be two complex conjugate eigenvalues of $A(t)$ for $t \in\left[0, t_{0}\right]$. Assume that (5) holds. Let every solution $\mathbf{x}$ of system (1) satisfies (37). If

$$
\|A(t)\| e^{-\int_{t}^{t_{0}} \operatorname{Re} \lambda(s) d s} \in L^{1}\left(0, t_{0}\right),
$$

then the zero solution of (1) is rectifiable attractive as $t \rightarrow 0$.

Proof. It is clear that the attractivity of the zero solution of system (1) follows from (5), (21), and (37). Next, by taking the matrix norm in (1) and using a priori estimate (37), we obtain,

$$
\begin{aligned}
\left\|\mathbf{x}^{\prime}(t)\right\|_{2} & =\|A(t) \mathbf{x}(t)\|_{2} \\
& \leq\|A(t)\|\|\mathbf{x}(t)\|_{2} \leq c\|A(t)\| e^{-\int_{t}^{t_{0}} \operatorname{Re} \lambda(s) d s} .
\end{aligned}
$$

Now, from the assumption (47) and the inequality (48), we observe that

$$
\begin{aligned}
& \lim _{\varepsilon \rightarrow 0} \int_{\varepsilon}^{t_{0}}\left\|\mathbf{x}^{\prime}(t)\right\|_{2} d t \\
& \quad \leq \lim _{\varepsilon \rightarrow 0} c \int_{\varepsilon}^{t_{0}}\|A(t)\| e^{-\int_{t}^{t_{0}} \operatorname{Re} \lambda(s) d s} d t<\infty .
\end{aligned}
$$

Hence, Lemma 9 proves this theorem.

If $A(t)$ admits the asymptotic behaviour given in (4), then a priori estimate (37) is fulfilled because of the asymptotic formula (21) and thus, the rectifiable attractivity of the zero solution of system (1), in such a case, holds without supposing (37), as follows.

Theorem 11. Let (4) be satisfied, and let $\lambda(t)=\operatorname{Re} \lambda(t) \pm$ $i \operatorname{Im} \lambda(t)$ be two complex conjugate eigenvalues of $A(t)$ for $t \epsilon$ $\left[0, t_{0}\right]$. Assume that (5) holds. If the condition (47) is fulfilled, then the zero solution of linear system (1) is rectifiable attractive as $t \rightarrow 0$.

Proof. It is clear that all assumptions of Theorem 11 ensure that Lemma 7 can be applied. Hence, by Lemma 7, that is, by asymptotic formula (21), one can easily show that a priori estimate (37) holds for all solutions $\mathbf{x}$ of system (1). Thus, all assumptions of Theorem 10 are fulfilled. Now, Theorem 10 proves Theorem 11. 


\section{Nonrectifiable Attractivity of the Zero Solution}

In this section we study the nonrectifiable attractivity for linear system (1). Unlike the rectifiable attractivity, the nonrectifiable attractivity is a more difficult case. It is because the required property $\mathbf{x}^{\prime} \notin L^{1}\left(0, t_{0}\right)$ cannot be derived from system (1) and therefore, we are not in an opportunity to use Lemma 9. Hence, instead of Lemma 9, we state and prove the next new lemma, which plays an essential role in the proof of the main results of this section.

Lemma 12. Let $u=u(t), u \in C\left(\left[0, t_{0}\right]\right)$, and let there be $a$ sequence $s_{n} \in\left(0, t_{0}\right]$, a number $n_{0} \in \mathbb{N}$, and two functions $\varphi=\varphi(t), a=a(t), t \in\left(0, t_{0}\right]$, such that

$\left(H_{1}\right)$ sequence $s_{n}$ is decreasing, $s_{n} \rightarrow 0$ as $n \rightarrow \infty$ and $u\left(s_{n}\right) u\left(s_{n+1}\right)<0$ for all $n \geq n_{0} ;$

$\left(H_{2}\right)$ function $\varphi(t), \varphi \in C^{1}\left(\left(0, t_{0}\right]\right)$, is strictly monotone near $t=0$, that is, $\varphi^{\prime}(t) \neq 0$ near $t=0$ and there is a constant $c_{2}>0$ such that

$$
\left|\varphi\left(s_{n+1}\right)-\varphi\left(s_{n}\right)\right| \leq c_{2} \quad \forall n \geq n_{0} ;
$$

$\left(H_{3}\right)$ function $a(t), a \in C\left(\left[0, t_{0}\right]\right)$, is nondecreasing and there is a constant $c_{1}>0$ such that

$$
\left|u\left(s_{n}\right)\right| \geq c_{1} a\left(s_{n}\right) \quad \forall n \geq n_{0} ;
$$

$\left(H_{4}\right) \lim _{\varepsilon \rightarrow 0} \int_{\varepsilon}^{t_{0}} a(t)\left|\varphi^{\prime}(t)\right| d t=\infty$.

Then the graph

$$
\Gamma_{u}=\left\{(x, y) \in \mathbb{R}^{2}: x=t, y=u(t) \text { for } t \in\left[0, t_{0}\right]\right\}
$$

of function $u(t)$ is a nonrectifiable curve in $\mathbb{R}^{2}$.

Proof. Because of $\left(H_{1}\right)$, there is a sequence $a_{n} \in\left(s_{n+1}, s_{n}\right)$ such that $u\left(a_{n}\right)=0$ for all $n \geq n_{0}$ and hence we have

$$
\begin{gathered}
0<\cdots<s_{n+1}<a_{n}<s_{n}<a_{n-1} \\
<\cdots<s_{n_{0}+1}<a_{n_{0}}<s_{n_{0}}<t_{0} .
\end{gathered}
$$

Let $t_{n} \in\left(0, t_{0}\right]$ be a sequence defined by

$$
t_{2 n-1}:=s_{n}, \quad t_{2 n}:=a_{n} \text { for } n \geq 1 .
$$

Obviously for all $m \geq n_{0}$, the set $\left\{0, t_{2 m-1}, \ldots, t_{2 n_{0}-1}, t_{0}\right\}$ makes a finite partition of interval $\left[0, t_{0}\right]$ and since length $\left(\Gamma_{u}\right)$ is a supremum of all sums $\sum\left|u\left(\tau_{n}\right)-u\left(\tau_{n-1}\right)\right|$ taken over all finite partitions $\left\{\tau_{n}\right\}$ of $\left[0, t_{0}\right]$; we conclude that

$$
\begin{aligned}
\text { length }\left(\Gamma_{u}\right) \geq & \left|u(0)-u\left(t_{2 m-1}\right)\right|+\sum_{n=2 n_{0}}^{2 m-1}\left|u\left(t_{n}\right)-u\left(t_{n-1}\right)\right| \\
& +\left|u\left(t_{2 n_{0}-1}\right)-u\left(t_{0}\right)\right| \\
\geq & \sum_{n=2 n_{0}}^{2 m-1}\left|u\left(t_{n}\right)-u\left(t_{n-1}\right)\right|=\sum_{n=n_{0}}^{m}\left|u\left(s_{n}\right)\right| .
\end{aligned}
$$

Next, let, for instance, $\varphi(t)$ be strictly decreasing near $t=0$ (the case when $\varphi(t)$ is strictly increasing could be analogously considered). Hence, from $\left(\mathrm{H}_{2}\right)$ and $\left(\mathrm{H}_{3}\right)$, it follows that

$$
\begin{aligned}
\sum_{n=n_{0}}^{m}\left|u\left(s_{n}\right)\right| & \geq c_{1} \sum_{n=n_{0}}^{m} a\left(s_{n}\right) \\
& \geq \frac{c_{1}}{c_{2}} \sum_{n=n_{0}}^{m} a\left(s_{n}\right)\left(\varphi\left(s_{n+1}\right)-\varphi\left(s_{n}\right)\right) \\
& =\frac{c_{1}}{c_{2}} \sum_{n=n_{0}}^{m} \int_{\varphi\left(s_{n}\right)}^{\varphi\left(s_{n+1}\right)} a\left(s_{n}\right) d \tau \\
& =\frac{c_{1}}{c_{2}} \sum_{n=n_{0}}^{m} \int_{\varphi\left(s_{n}\right)}^{\varphi\left(s_{n+1}\right)}\left[\max _{t \in\left[s_{n+1}, s_{n}\right]} a(t)\right] d \tau \\
& \geq \frac{c_{1}}{c_{2}} \sum_{n=n_{0}}^{m} \int_{\varphi\left(s_{n}\right)}^{\varphi\left(s_{n+1}\right)} a\left(\varphi^{-1}(\tau)\right) d \tau \\
& =\frac{c_{1}}{c_{2}} \sum_{n=n_{0}}^{m} \int_{s_{n+1}}^{s_{n}} a(t)\left|\varphi^{\prime}(t)\right| d t .
\end{aligned}
$$

Now from (55) and previous inequality, for all $m \geq n_{0}$ we obtain

$$
\begin{aligned}
\text { length }\left(\Gamma_{u}\right) & \geq \frac{c_{1}}{c_{2}} \sum_{n=n_{0}}^{m} \int_{s_{n+1}}^{s_{n}} a(t)\left|\varphi^{\prime}(t)\right| d t \\
& =\frac{c_{1}}{c_{2}} \int_{s_{m+1}}^{s_{n_{0}}} a(t)\left|\varphi^{\prime}(t)\right| d t .
\end{aligned}
$$

Letting $m \rightarrow \infty$ in previous inequality, the hypothesis $\left(H_{4}\right)$ proves this lemma.

Now, we are able to prove the following result.

Theorem 13. Let $\lambda(t)=\operatorname{Re} \lambda(t) \pm i \operatorname{Im} \lambda(t)$ be two complex conjugate eigenvalues of $A(t)$ for $t \in\left(0, t_{0}\right]$. Assume that $\operatorname{Re} \lambda(t) \geq 0$ and $\operatorname{Im} \lambda(t) \neq 0$ near $t=0$ and that (5) holds. Let every solution $\mathbf{x}(t)=(x(t), y(t))$ of system (1) satisfy (37) as well as the following assumption: let $u(t)$ be defined by $u(t):=x(t)$ or $u(t):=y(t)$ and let there be two constants $c_{1}>0, c_{2}>0$, a number $n_{0} \in \mathbb{N}$, and a decreasing sequence $s_{n} \in\left(0, t_{0}\right], s_{n} \rightarrow 0$ as $n \rightarrow \infty$, which all depend on function $u(t)$, such that for all $n \geq n_{0}$, one has

$$
\begin{gathered}
u\left(s_{n}\right) u\left(s_{n+1}\right)<0, \quad\left|u\left(s_{n}\right)\right| \geq c_{1} e^{-\int_{s_{n}}^{t_{0}} \operatorname{Re} \lambda(t) d t}, \\
\left|\int_{s_{n+1}}^{s_{n}} \operatorname{Im} \lambda(t) d t\right| \leq c_{2} .
\end{gathered}
$$

If

$$
\operatorname{Im} \lambda(t) e^{-\int_{t}^{t_{0}} \operatorname{Re} \lambda(s) d s} d t \notin L^{1}\left(0, t_{0}\right),
$$

then the zero solution of (1) is nonrectifiable attractive as $t \rightarrow$ 0 . 
Proof. At first, the attractivity of the zero solution of linear system (1) follows from the assumptions (5) and (37).

Next, let $\varphi(t)$ and $a(t)$ denote two new functions defined by

$$
\varphi(t)=\int_{t}^{t_{0}} \operatorname{Im} \lambda(s) d s, \quad a(t)=e^{-\int_{t}^{t_{0}} \operatorname{Re} \lambda(s) d s} .
$$

Let $u(t):=x(t)$ or $u(t):=y(t)$, where $\mathbf{x}(t)=(x(t), y(t))$ is a solution of system (1). With the help of notation (60) and assumption (58), it is easy to check that the function $u(t)$ satisfies all hypotheses $\left(H_{1}\right),\left(H_{2}\right)$, and $\left(H_{3}\right)$ from Lemma 12 . Indeed, the existence of a sequence $s_{n} \in\left(0, t_{0}\right)$ satisfying $\left(H_{1}\right)$ follows from the first inequality in (58). Also, from (18), (58), and (60) we obtain

$$
\begin{aligned}
& \varphi^{\prime}(t)=-\operatorname{Im} \lambda(t) \neq 0 \quad \text { near } t=0, \\
& \left|\varphi\left(s_{n+1}\right)-\varphi\left(s_{n}\right)\right|=\left|\int_{s_{n}}^{s_{n+1}} \operatorname{Im} \lambda(s) d s\right| \leq c_{2} \quad \forall n \geq n_{0}, \\
& \left|u\left(s_{n}\right)\right| \geq c_{1} e^{-\int_{s_{n}}^{t_{0}} \operatorname{Re} \lambda(s) d s}=c_{1} a\left(s_{n}\right) \quad \forall n \geq n_{0},
\end{aligned}
$$

which show that $\left(\mathrm{H}_{2}\right)$ and $\left(\mathrm{H}_{3}\right)$ from Lemma 12 are fulfilled too. Moreover, the equality

$$
a(t)\left|\varphi^{\prime}(t)\right|=|\operatorname{Im} \lambda(t)| e^{-\int_{t}^{t_{0}} \operatorname{Re} \lambda(s) d s}
$$

together with assumption (59) ensure that the hypothesis $\left(H_{4}\right)$ from Lemma 12 is also fulfilled. Now, by Lemma 12 we obtain that the graph of $u(t):=x(t)$ or $u(t):=y(t)$ is a nonrectifiable curve in $\mathbb{R}^{2}$. In particular, it follows that the solution curve $\Gamma_{\mathbf{x}}$ is also a nonrectifiable curve in $\mathbb{R}^{2}$, where $\mathbf{x}(t)=(x(t), y(t))$.

In the next theorem, the nonrectifiable attractivity is obtained without supposing the a priori estimates (37) and (58), since they immediately follow from the asymptotic behaviour of $A(t)$ near $t=0$ given in (4).

Theorem 14. Let (4) be satisfied, and let $\lambda(t)=\operatorname{Re} \lambda(t) \pm$ $i \operatorname{Im} \lambda(t)$ be two complex conjugate eigenvalues of the matrix $A(t)$ for $t \in\left(0, t_{0}\right]$. Assume that $\operatorname{Re} \lambda(t) \geq 0$ near $t=0$ and that (5) holds. If the condition (59) holds, then the zero solution of (1) is nonrectifiable attractive as $t \rightarrow 0$.

Proof. This proof is a consequence of Theorem 13 and the asymptotic formula (21). At first, according to Lemma 7, every solution $\mathbf{x}(t)=(x(t), y(t))$ of system (1) satisfies (21). If we denote by $u(t):=x(t)$ or $u(t):=y(t)$, then (21) ensures that $u(t)$ could be written in the form

$$
\begin{aligned}
u(t)=[ & \left.c_{1}+\varepsilon_{1}(t)\right] a(t) \cos \varphi(t) \\
& +\left[c_{2}+\varepsilon_{2}(t)\right] a(t) \sin \varphi(t) \quad \text { near } t=0
\end{aligned}
$$

where $c_{1}, c_{2} \in \mathbb{R}$ with $c_{1}^{2}+c_{2}^{2}>0, \varepsilon_{1}, \varepsilon_{2} \in C\left(0, t_{0}\right]$, $\lim _{t \rightarrow 0} \varepsilon_{1}(t)=\lim _{t \rightarrow 0} \varepsilon_{2}(t)=0$, and $a(t), \varphi(t)$ are defined in $(60)$.
Since $\lambda(t)$ is a continuous complex-valued function on $\left(0, t_{0}\right]$, from $(18)$, we conclude that

either $\operatorname{Im} \lambda(t)<0$ or $\operatorname{Im} \lambda(t)>0$ near $t=0$.

Therefore, there are two real constants $c_{3}, c_{4} \in \mathbb{R}$ and $\delta \in$ $C\left(0, t_{0}\right]$ such that $c_{3} \neq 0, \lim _{t \rightarrow 0} \delta(t)=0$ and

$$
\begin{aligned}
u(t)= & {\left[c_{3}+\delta(t)\right] e^{-\int_{t}^{t_{0}} \operatorname{Re} \lambda(\sigma) d \sigma} } \\
& \times \sin \left(\int_{t}^{t_{0}}|\operatorname{Im} \lambda(\sigma)| d \sigma+c_{4}\right) \quad \text { near } t=0 .
\end{aligned}
$$

Because of (59), we have $\int_{t}^{t_{0}}|\operatorname{Im} \lambda(\sigma)| d \sigma \rightarrow \infty$ as $t \rightarrow$ 0 and therefore there is a decreasing sequence $s_{n} \in\left(0, t_{0}\right]$ and a number $n_{0} \in \mathbb{N}$ such that

$$
\int_{s_{n}}^{t_{0}}|\operatorname{Im} \lambda(\sigma)| d \sigma+c_{4}=\frac{\pi}{2}+n \pi \quad \forall n \geq n_{0} .
$$

Putting (66) into (65) and retaking $n_{0} \in \mathbb{N}$ as sufficiently large, we get

$$
\begin{aligned}
& u\left(s_{n}\right) u\left(s_{n+1}\right)<0, \\
& \left|u\left(s_{n}\right)\right| \geq \frac{\left|c_{3}\right|}{2} e^{-\int_{s_{n}}^{t_{0}} \operatorname{Re} \lambda(s) d s} \quad \forall n \geq n_{0},
\end{aligned}
$$

and because of (64) and (66),

$$
\begin{aligned}
\left|\int_{s_{n+1}}^{s_{n}} \operatorname{Im} \lambda(s) d s\right| & =\int_{s_{n+1}}^{s_{n}}|\operatorname{Im} \lambda(s)| d s \\
& =\int_{s_{n+1}}^{t_{0}}|\operatorname{Im} \lambda(s)| d s \\
& -\int_{s_{n}}^{t_{0}}|\operatorname{Im} \lambda(s)| d s=\pi .
\end{aligned}
$$

Now, statements (67) and (68) show that the function $u(t)$ and sequence $s_{n}$ defined, respectively, in (65) and (66) satisfy the required condition (58). Therefore, the main conclusion of this theorem immediately follows from Theorem 13.

\section{Proof of Theorem 4}

In this section, we prove the rectifiable and nonrectifiable attractivity for the zero solution of model system (7) as a direct consequence of Theorem 3 .

Let $A(t)$ be the matrix of the model system (7) given by

$$
A(t)=\left(\begin{array}{cc}
e(t) t^{-p} & f(t) t^{-2} \\
-g(t) t^{-2} & h(t) t^{-q}
\end{array}\right) \quad \text { for } t \in\left(0, t_{0}\right]
$$

where the real numbers $p, q$, and functions $e, f, g$, and $h$ satisfy (8), (9), and (10), respectively. We will show that such a matrix $A(t)$ together with its eigenvalues $\lambda(t)$ satisfy the required conditions $\operatorname{Re} \lambda(t) \geq 0$ near $t=0$, (5), and (4).

At first, because of (9), we have $\operatorname{Re} \lambda(t)=\left(e(t) t^{-p}+\right.$ $\left.h(t) t^{-q}\right) / 2>0$. 
Next, since $\max \{p, q\} \geq 1$, let, for instance, $\max \{p, q\}=$ $p \geq 1$, and $t_{0} \in(0,1]$; from (9) we obtain a constant $c_{0}>0$ such that

$$
\begin{aligned}
\int_{t}^{t_{0}} \operatorname{Re} \lambda(s) d s & =\frac{1}{2} \int_{t}^{t_{0}}\left(e(s) s^{-p}+h(s) s^{-q}\right) d s \\
& \geq \frac{1}{2} \int_{t}^{t_{0}} e(s) s^{-p} d s \\
& \geq c_{0} \int_{t}^{t_{0}} s^{-1} d s=c_{0} \ln \frac{t_{0}}{t} \longrightarrow \infty \quad \text { as } t \longrightarrow 0
\end{aligned}
$$

Now, it is clear that (70) proves the desired statement (5).

Finally, since $p<2$ and $q<2$,

$$
\begin{aligned}
\lim _{t \rightarrow 0}\left[t^{2} A(t)\right] & =\lim _{t \rightarrow 0}\left(\begin{array}{cc}
e(t) t^{2-p} & f(t) \\
-g(t) & h(t) t^{2-q}
\end{array}\right) \\
& =\left(\begin{array}{cc}
0 & f(0) \\
-g(0) & 0
\end{array}\right)=: A_{0},
\end{aligned}
$$

where $A_{0}$ is a constant matrix. Since $f(0) g(0)>0$, matrix $A_{0}$ has two different eigenvalues. So, the condition (4) is fulfilled too.

Thus, matrix $A(t)$ together with its eigenvalues satisfy all required conditions so that Theorem 3 can be applied to the model system (7) in all cases of $p$ and $q$ given in (11)(16). Since by Theorem 3 the rectifiable and nonrectifiable attractivity of the zero solution of (1) depends on the integrability of function

$$
\Phi(t)=|\operatorname{Im} \lambda(t)| e^{-\int_{t}^{t_{0}} \operatorname{Re} \lambda(s) d s} \quad \text { for } t \in\left(0, t_{0}\right],
$$

we can conclude that the zero solutions of (7) is rectifiable attractive if $\Phi \in L^{1}\left(0, t_{0}\right)$ and nonrectifiable attractive if $\Phi \notin$ $L^{1}\left(0, t_{0}\right)$. Let us remark that in Lemma 6 , it has been proved that

$$
|\operatorname{Im} \lambda(t)| \sim t^{-2} \quad \text { as } t \longrightarrow 0
$$

(i) Assume that $\max \{p, q\}>1$. For instance, we assume $p>1$. Since $e(t)>0$ on $\left[0, t_{0}\right]$, we have $e(t) t^{-p+1} \rightarrow \infty$ as $t \rightarrow 0$. In particular, there exist $t_{1} \in\left(0, t_{0}\right]$ and $\delta>0$ such that

$$
e(t) t^{-p+1} \geq 2+\delta \quad \text { for } t \in\left(0, t_{1}\right]
$$

Since

$$
e^{-\int_{t}^{t_{0}} \operatorname{Re} \lambda(s) d s}=e^{(-1 / 2) \int_{t}^{t_{0}}\left(e(s) s^{-p}+h(s) s^{-q}\right) d s},
$$

we have

$$
\begin{aligned}
e^{-\int_{t}^{t_{0}} \operatorname{Re} \lambda(s) d s} & \leq e^{(-1 / 2) \int_{t}^{t_{0}} e(s) s^{-p} d s} \\
& =e^{(-1 / 2) \int_{t}^{t_{0}} e(s) s^{-p+1} s^{-1} d s} \\
& \leq e^{-(1+(\delta / 2)) \int_{t}^{t_{0}} s^{-1} d s}=c t^{1+(\delta / 2)},
\end{aligned}
$$

where the constant $c=t_{0}^{-1-(\delta / 2)}$. Now from (72), (73), and (76), we observe that

$$
\Phi(t) \leq c_{1} t^{-2} t^{1+(\delta / 2)}=c_{1} t^{-1+(\delta / 2)} \in L^{1}\left(0, t_{0}\right) .
$$

Thus, it is shown that $\Phi \in L^{1}\left(0, t_{0}\right)$.

(ii) Assume that (11) is fulfilled. Since $e(t)>2$ on $\left[0, t_{0}\right]$, there exists $\delta>0$ such that $e(t) \geq 2+\delta$ on $\left[0, t_{0}\right]$. Since $p=1$ and $h(t)>0$ on $\left(0, t_{0}\right]$, from (75) we have

$$
\begin{aligned}
e^{-\int_{t}^{t_{0}} \operatorname{Re} \lambda(s) d s} & \leq e^{(-1 / 2) \int_{t}^{t_{0}} e(s) s^{-1} d s} \\
& \leq e^{-(1+(\delta / 2)) \int_{t}^{t_{1}} s^{-1} d s}=c t^{1+(\delta / 2)},
\end{aligned}
$$

where the constant $c=t_{0}^{-1-(\delta / 2)}$. Now from (72), (73), and (78), we obtain $\Phi \in L^{1}\left(0, t_{0}\right)$. When (12) is fulfilled, we obtain $\Phi \in L^{1}\left(0, t_{0}\right)$ by the similar argument.

Assume that (13) is fulfilled. Since $e(t)+h(t)>2$ on $\left[0, t_{0}\right]$, there exists $\delta>0$ such that $e(t)+h(t) \geq 2+\delta$ on $\left[0, t_{0}\right]$. From (75) we have

$$
\begin{aligned}
e^{-\int_{t}^{t_{0}} \operatorname{Re} \lambda(s) d s} & =e^{(-1 / 2) \int_{t}^{t_{0}} e(s)+h(s) s^{-1} d s} \\
& \leq e^{-(1+(\delta / 2)) \int_{t}^{t_{0}} s^{-1} d s}=c t^{1+(\delta / 2)},
\end{aligned}
$$

where the constant $c=t_{0}^{-1-(\delta / 2)}$. Thus we obtain $\Phi \in L^{1}\left(0, t_{0}\right)$ by the argument above.

(iii) Let (14) be fulfilled. Since $h \in C\left(\left[0, t_{0}\right]\right)$, there is a constant $m_{0}>0$ such that $h(t) \leq m_{0}$ on $\left[0, t_{0}\right]$, and since $q<1$ there is a constant $c_{0}>0$ such that

$$
\begin{array}{r}
e^{(-1 / 2) \int_{t}^{t_{0}} h(s) s^{-q} d s} \geq e^{-\left(m_{0} / 2\right) \int_{t}^{t_{0}} s^{-q} d s} \geq \mathcal{c}_{0} \\
\text { as } t \longrightarrow 0 .
\end{array}
$$

Hence,

$$
\begin{aligned}
e^{-\int_{t}^{t_{0}} \operatorname{Re} \lambda(s) d s} & =e^{(-1 / 2) \int_{t}^{t_{0}}\left(e(s) s^{-1}+h(s) s^{-q}\right) d s} \\
& =e^{-\int_{t}^{t_{0}} s^{-1} d s} e^{(-1 / 2) \int_{t}^{t_{0}} h(s) s^{-q} d s} \\
& \geq c_{0} e^{-\int_{t}^{t_{0}} s^{-1} d s}=c_{0} \frac{t}{t_{0}} .
\end{aligned}
$$

Now, by combining (72), (73), and (81), we observe

$$
\begin{array}{r}
\int_{t}^{t_{0}} \Phi(s) d s \geq \frac{c_{1}}{t_{0}} \int_{t}^{t_{0}} s^{-2} s d s=\frac{c_{1}}{t_{0}} \ln \frac{t_{0}}{t} \\
\longrightarrow \infty \\
\text { as } t \rightarrow 0 .
\end{array}
$$

Thus, $\Phi \notin L^{1}\left(0, t_{0}\right)$. When (15) is fulfilled, we similarly obtain $\Phi \notin L^{1}\left(0, t_{0}\right)$.

Let (16) be fulfilled. Then, from (75) we have

$$
\begin{aligned}
e^{-\int_{t}^{t_{0}} \operatorname{Re} \lambda(s) d s} & =e^{(-1 / 2) \int_{t}^{t_{0}}(e(s)+h(s)) s^{-1} d s} \\
& \geq e^{-\int_{t}^{t_{0}} s^{-1} d s}=\frac{t}{t_{0}} .
\end{aligned}
$$

By combining (72), (73), and (83), we obtain $\Phi \notin L^{1}\left(0, t_{0}\right)$. Thus, the proof is completed. 


\section{Appendix}

At first, we state and prove the existence and uniqueness of solutions of linear system (1).

Theorem A.1. There exists a unique solution $\mathbf{x}$ of linear system (1).

Proof. Putting $\tau=1 / t$ into system (1) and denoting $\mathbf{w}(\tau)=$ $\mathbf{x}(1 / \tau)$, we obtain the equivalent system

$$
\frac{d \mathbf{w}}{d \tau}=B(\tau) \mathbf{w} \quad \text { for } \tau \in\left[\tau_{0}, \infty\right), \quad \mathbf{w}\left(\tau_{0}\right)=\mathbf{x}_{0},
$$

where $\tau_{0}=1 / t_{0}$ and the matrix $B(t)$ is defined by (23). By the standard theory for system (A.1), we obtain the existence and uniqueness of the solution to (1).

At the second, we give some simple sufficient conditions on the matrix-valued function $A=A(t)$ such that the solution curve $\Gamma_{\mathbf{x}}$ of every nontrivial solution $\mathbf{x}(t)$ of linear system (1) (as well as of (7)) is a Jordan curve.

Definition A.2. A solution $\mathbf{x}$ of linear system (1) is said to be trivial solution of (1) near $t=0$ if there is $t_{1} \in\left(0, t_{0}\right]$ such that $\mathbf{x}(t)=0$ for all $t \in\left(0, t_{1}\right]$. Otherwise, $\mathbf{x}(t)$ is said to be nontrivial solution of (1) near $t=0$.

Theorem A.3. Let the matrix elements $a_{i, j}(t)$ of $A(t)$ satisfy

$$
\begin{aligned}
\min & \left\{a_{1,1}(t), a_{2,2}(t)\right\} \\
> & \frac{1}{2}\left|a_{1,2}(t)+a_{2,1}(t)\right| \quad t \in\left(0, t_{0}\right] .
\end{aligned}
$$

If $\mathbf{x}$ is a nontrivial solution of linear system (1) near $t=0$, then $\|\mathbf{x}(t)\|_{2}$ is strictly positive and decreasing on $\left(0, t_{0}\right]$. In particular, the solution curve $\Gamma_{\mathbf{x}}$ is a Jordan curve.

Remark A.4. It is clear that if $a_{1,1}(t)>0, a_{2,2}(t)>0$ and $a_{1,2}(t)=-a_{2,1}(t)$ on $\left(0, t_{0}\right]$, then condition (A.2) is fulfilled.

Proof of Theorem A.3. Let $\mathbf{x}(t)=(x(t), y(t))$ be a nontrivial solution of system (1) near $t=0$. The assumption (A.2) can be rewritten as

$$
\begin{aligned}
& a_{1,1}(t)>\frac{1}{2}\left|a_{1,2}(t)+a_{2,1}(t)\right| \\
& a_{2,2}(t)>\frac{1}{2}\left|a_{1,2}(t)+a_{2,1}(t)\right| \quad \text { on }\left(0, t_{0}\right] .
\end{aligned}
$$

Let $v(t)$ be a function defined by

$$
v(t)=\|\mathbf{x}(t)\|^{2}=x^{2}(t)+y^{2}(t) \quad \text { for } t \in\left(0, t_{0}\right] .
$$

Taking the derivative $d / d t$ in (A.4), it follows that

$$
\frac{d v}{d t}=2 x(t) x^{\prime}(t)+2 y(t) y^{\prime}(t) \quad \text { for } t \in\left(0, t_{0}\right]
$$

Multiplying the first and second equation in system (1), respectively, by $x(t)$ and $y(t)$, we obtain

$$
\begin{aligned}
x(t) & x^{\prime}(t)+y(t) y^{\prime}(t) \\
= & a_{1,1}(t) x^{2}(t)+\left(a_{1,2}(t)+a_{2,1}(t)\right) x(t) y(t) \\
& +a_{2,2}(t) y^{2}(t) .
\end{aligned}
$$

Because of inequality $a b \leq\left(a^{2}+b^{2}\right) / 2$, we have

$$
\begin{aligned}
& \left|\left(a_{1,2}(t)+a_{2,1}(t)\right) x(t) y(t)\right| \\
& \quad \leq \frac{1}{2}\left|a_{1,2}(t)+a_{2,1}(t)\right|\left(x^{2}(t)+y^{2}(t)\right),
\end{aligned}
$$

which together with (A.6) derives that

$$
\begin{aligned}
x(t) & x^{\prime}(t)+y(t) y^{\prime}(t) \\
\geq & a_{1,1}(t) x^{2}(t)-\frac{1}{2}\left|a_{1,2}(t)+a_{2,1}(t)\right|\left(x^{2}(t)+y^{2}(t)\right) \\
& +a_{2,2}(t) y^{2}(t)=D(t),
\end{aligned}
$$

where $D(t)$ denotes the function

$$
\begin{aligned}
D(t)= & \left(a_{1,1}(t)-\frac{1}{2}\left|a_{1,2}(t)+a_{2,1}(t)\right|\right) x^{2}(t) \\
& +\left(a_{2,2}(t)-\frac{1}{2}\left|a_{1,2}(t)+a_{2,1}(t)\right|\right) y^{2}(t) .
\end{aligned}
$$

Putting (A.8) into (A.5), we obtain

$$
\frac{d v}{d t} \geq 2 D(t) \quad \text { for } t \in\left(0, t_{0}\right] .
$$

Because of (A.3), we have $D(t) \geq 0$ on $\left(0, t_{0}\right]$. Hence, from (A.10), we observe $d v / d t \geq 0$ on $\left(0, t_{0}\right]$. Integrating this inequality over the interval $[s, t]$ for all $0<s \leq t \leq t_{0}$, we get $v(s) \leq v(t)$. If we suppose for a moment that $v(t)=0$, then from previous inequality we get $v(s)=0$ for all $s \in(0, t]$, which is not possible because of Definition A.2. Therefore, $v(t)>0$ for all $t \in\left(0, t_{0}\right]$, which implies $\|\mathbf{x}(t)\|_{2}>0$ for all $t \in\left(0, t_{0}\right]$ because of (A.4). It proves the first statement of this theorem.

Next, with the help of (A.3) and $\|\mathbf{x}(t)\|_{2}>0$ for all $t \epsilon$ $\left(0, t_{0}\right]$, we deduce that $D(t)>0$ on $\left(0, t_{0}\right]$, which together with (A.10) gives $v^{\prime}(t)>0$ on $\left(0, t_{0}\right]$. Integrating this inequality over the interval $[s, t]$ for all $0<s<t \leq t_{0}$, we get

$$
v(s)<v(t) \quad \forall s, t \text { such that } 0<s<t \leq t_{0} .
$$

If, for instance, there exist $s, t$ such that $0<s<t \leq t_{0}$ and $\|\mathbf{x}(s)\|_{2}=\|\mathbf{x}(t)\|_{2}$, then involving this equality into (A.4), we obtain $v(s)=v(t)$, which is not possible because of (A.11). Therefore, $\|\mathbf{x}(t)\|_{2} \neq\|\mathbf{x}(s)\|_{2}$. Thus, it is shown that $\Gamma_{\mathbf{x}}$ is a Jordan curve near $t=0$. In the same way, $\|\mathbf{x}(s)\|_{2}<\|\mathbf{x}(t)\|_{2}$ for all $s, t$ such that $0<s<t \leq t_{0}$.

According to Remark A.4, previous theorem can be applied to model system (7). 
Corollary A.5. Let $e, h \in C\left(\left(0, t_{0}\right]\right), e(t)>0, h(t)>0$, and $f(t)=g(t)$ near $t=0$. If $\mathbf{x}(t)$ is a nontrivial solution of linear system (7) near $t=0$, then $\|\mathbf{x}(t)\|_{2}$ is strictly positive and decreasing on $\left(0, t_{0}\right]$. In particular, the solution curve $\Gamma_{\mathbf{x}}$ is a Jordan curve.

Finally, we point out that in some essential cases, the model system (7) allows the explicit form of all its solutions. Such systems are called the integrable systems; for details, see [4]. Hence, the rectifiable and nonrectifiable attractivity of the zero solution of (7) in such cases can be reproven in an explicit way. That is, the statements of Theorem 4 can be confirmed once more, but explicitly.

Proposition A.6. Let $h(t)>0$ and $f(t)>0$ on $\left(0, t_{0}\right]$. The fundamental system of all solutions of linear differential system

$$
\begin{gathered}
x^{\prime}(t)=h(t) t^{-p} x(t)+f(t) t^{-2} y(t), \\
y^{\prime}(t)=-f(t) t^{-2} x(t)+h(t) t^{-p} y(t), \quad t \in\left(0, t_{0}\right]
\end{gathered}
$$

is explicitly given by the formula

$$
\begin{aligned}
x(t)= & e^{-\int_{t}^{t_{0}} h(s) s^{-p} d s} \\
\times & {\left[-c_{1} \sin \left(\int_{t}^{t_{0}} f(s) s^{-2} d s\right)\right.} \\
& \left.\quad+c_{2} \cos \left(\int_{t}^{t_{0}} f(s) s^{-2} d s\right)\right], \\
y(t)= & e^{-\int_{t}^{t_{0}} h(s) s^{-p} d s} \\
\times & {\left[c_{1} \cos \left(\int_{t}^{t_{0}} f(s) s^{-2} d s\right)\right.} \\
& \left.+c_{2} \sin \left(\int_{t}^{t_{0}} f(s) s^{-2} d s\right)\right] .
\end{aligned}
$$

Obviously for $p=q, e(t)=h(t)>0$ and $g(t)=f(t)>0$ on $\left(0, t_{0}\right]$, the system (A.12) is a particular case of our model system (7). Hence, according to Theorem 4 , we can state the rectifiable and nonrectifiable attractivity near $x=0$ for the system (A.12) as follows.

Corollary A.7. Let $h(t)>0$ and $f(t)>0$ on $\left(0, t_{0}\right]$.

(i) If $p>1$, then the zero solution of (A.12) is rectifiable attractive as $t \rightarrow 0$.

(ii) If $p=1$, then the zero solution of (A.12) is rectifiable attractive as $t \rightarrow 0$ provided that $h(t)>1$.

(iii) If $p=1$, then the zero solution of (A.12) is nonrectifiable attractive as $t \rightarrow 0$ provided that $h(t) \leq 1$.

However, since by Proposition A.6 the system (A.12) allows the explicit form of all its solutions, this means that Corollary A.7 can be also shown by using Lemma 9, Lemma 12, and Proposition A.6.

\section{References}

[1] K. Falconer, Fractal Geometry, Mathematical Foundations and Applications, John Wiley \& Sons, 1999.

[2] P. Mattila, Geometry of Sets and Measures in Euclidean Spaces, vol. 44 of Fractals and Rectifiability, Cambridge University Press, Cambridge, UK, 1995.

[3] C. Tricot, Curves and Fractal Dimension, Springer, New York, NY, USA, 1995.

[4] S. Miličić and M. Pašić, "Nonautonomous differential equations in Banach space and nonrectifiable attractivity in twodimensional linear differential systems," Abstract and Applied Analysis, vol. 2013, Article ID 935089, 10 pages, 2013.

[5] W. A. Coppel, Stability and Asymptotic Behavior of Differential Equations, D. C. Heath and Co., Boston, Mass, USA, 1965.

[6] L. Cesari, Asymptotic Behavior and Stability Problems in Ordinary Differential Equations, Springer, New York, NY, USA, 3rd edition, 1971.

[7] I. T. Kiguradze, Some Singular Boundary Value Problems for Ordinary Differential Equations, Izdatel'stvo Tbilisskogo Universiteta, Tbilisi, Ga, USA, 1975.

[8] P. Hartman, Ordinary Differential Equations, Birkhäuser, Boston, Mass, USA, 2nd edition, 1982.

[9] M. Pinto, "Asymptotic integration of second-order linear differential equations," Journal of Mathematical Analysis and Applications, vol. 111, no. 2, pp. 388-406, 1985.

[10] J. Rovder, "Asymptotic and oscillatory behaviour of solutions of a linear differential equation," Journal of Computational and Applied Mathematics, vol. 41, no. 1-2, pp. 41-47, 1992.

[11] S. Castillo and M. Pinto, "Asymptotic integration of ordinary different systems," Journal of Mathematical Analysis and Applications, vol. 218, no. 1, pp. 1-12, 1998.

[12] I. Rachunkova and I. Rachunek, "Asymptotic formula for oscillatory solutions of some singular nonlinear differential equation," Abstract and Applied Analysis, vol. 2011, Article ID 981401, 9 pages, 2011.

[13] J. Jaros and T. Kusano, "Existence and precise asymptotic behavior of strongly monotone solutions of systems of nonlinear differential equations," Difference Equations and Their Applications, vol. 5, pp. 185-204, 2013.

[14] M. Fečkan, Bifurcation and Chaos in Discontinuous and Continuous Systems, Nonlinear Physical Science, Springer, 2011.

[15] O. G. Mustafa and Y. V. Rogovchenko, "Asymptotic integration of a class of nonlinear differential equations," Applied Mathematics Letters, vol. 19, no. 9, pp. 849-853, 2006.

[16] M. Rasmussen, Attractivity and Bifurcation for Nonautonomous Dynamical Systems, vol. 1907 of Lecture Notes in Mathematics, Springer, Berlin, Germany, 2007.

[17] L. Hatvani, "On the asymptotic stability for a two-dimensional linear nonautonomous differential system," Nonlinear Analysis: Theory, Methods \& Applications, vol. 25, no. 9-10, pp. 991-1002, 1995.

[18] J. Sugie and M. Onitsuka, "Integral conditions on the uniform asymptotic stability for two-dimensional linear systems with time-varying coefficients," Proceedings of the American Mathematical Society, vol. 138, no. 7, pp. 2493-2503, 2010.

[19] F. Amato, M. Ariola, M. Carbone, and C. Cosentino, "Finitetime control of linear systems: a survey," in Current Trends in Nonlinear Systems and Control, pp. 195-213, Birkhäuser, Boston, Mass, USA, 2006. 
[20] G. Haller, "A variational theory of hyperbolic Lagrangian coherent structures," Physica D, vol. 240, no. 7, pp. 574-598, 2011.

[21] K. Rateitschak and O. Wolkenhauer, "Thresholds in transient dynamics of signal transduction pathways," Journal of Theoretical Biology, vol. 264, no. 2, pp. 334-346, 2010.

[22] M. K. Kwong, M. Pašić, and J. S. W. Wong, "Rectifiable oscillations in second-order linear differential equations," Journal of Differential Equations, vol. 245, no. 8, pp. 2333-2351, 2008.

[23] M. Pašić, "Rectifiable and unrectifiable oscillations for a class of second-order linear differential equations of Euler type," Journal of Mathematical Analysis and Applications, vol. 335, no. 1, pp. 724-738, 2007.

[24] M. Pašić and J. S. W. Wong, "Rectifiable oscillations in secondorder half-linear differential equations," Annali di Matematica Pura ed Applicata. Series IV, vol. 188, no. 3, pp. 517-541, 2009.

[25] M. Pašić and S. Tanaka, "Rectifiable oscillations of self-adjoint and damped linear differential equations of second-order," Journal of Mathematical Analysis and Applications, vol. 381, no. 1, pp. 27-42, 2011.

[26] J. S. W. Wong, "On rectifiable oscillation of Euler type second order linear differential equations," Electronic Journal of Qualitative Theory of Differential Equations, vol. 20, pp. 1-12, 2007.

[27] J. S. W. Wong, "On rectifiable oscillation of Emden-Fowler equations," Memoirs on Differential Equations and Mathematical Physics, vol. 42, pp. 127-144, 2007.

[28] L. C. Evans and R. F. Gariepy, Measure Theory and Fine Properties of Functions, CRC Press, New York, NY, USA, 1999. 


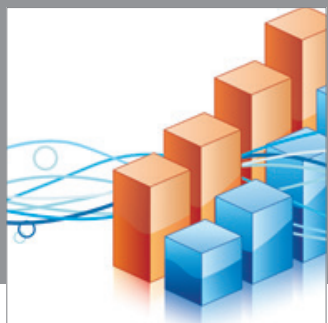

Advances in

Operations Research

mansans

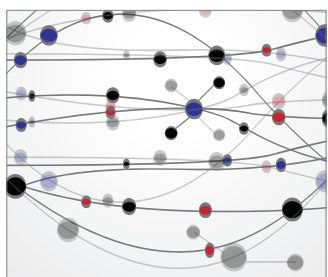

The Scientific World Journal
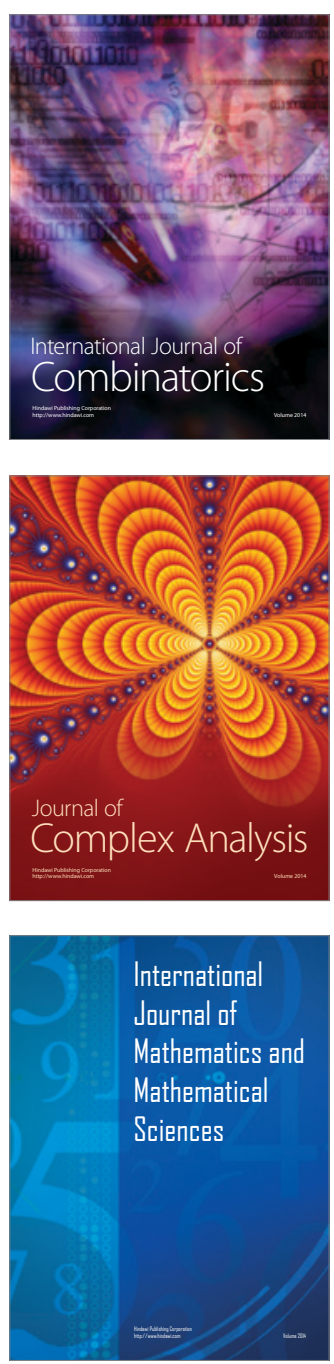
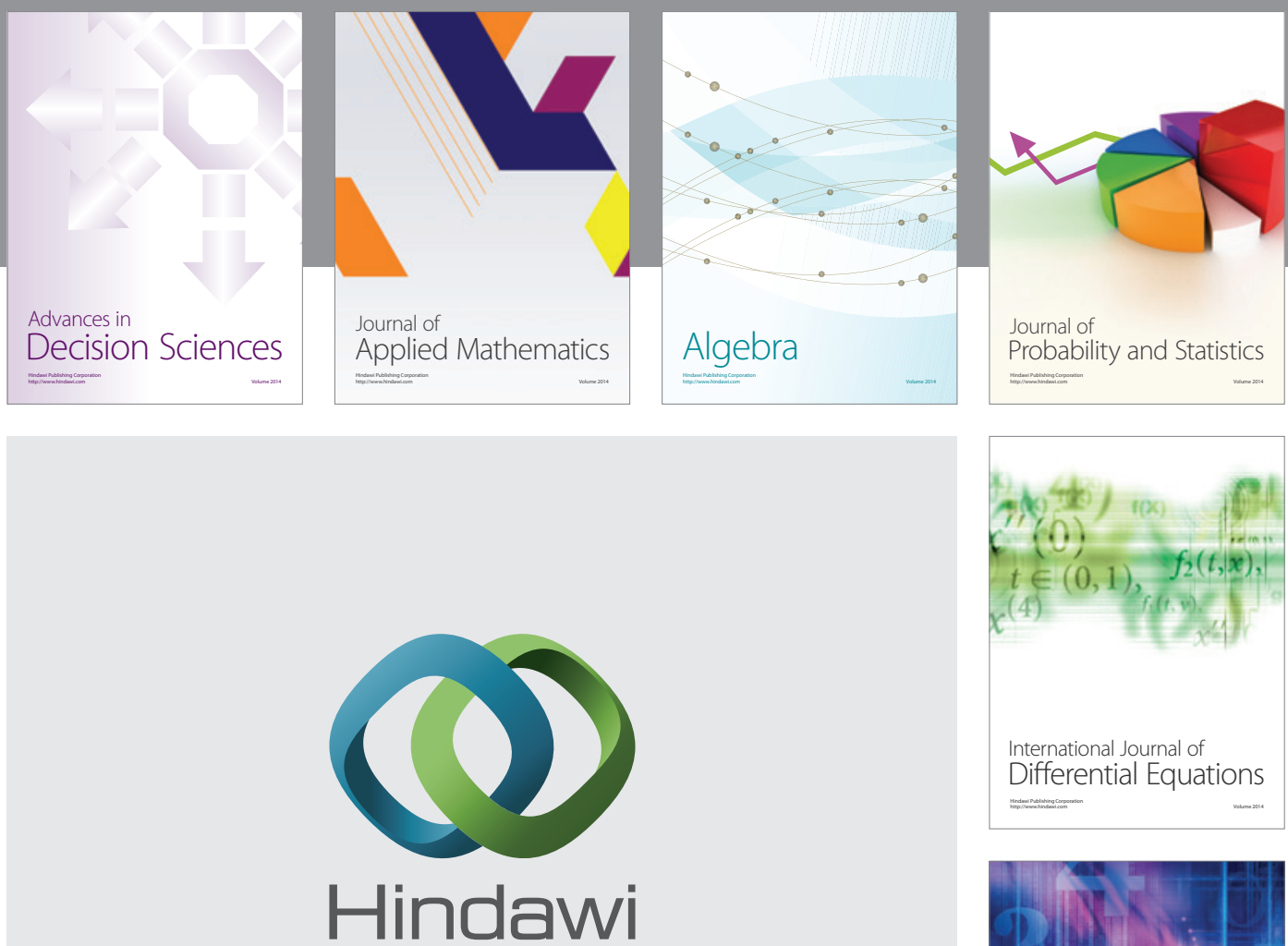

Submit your manuscripts at http://www.hindawi.com
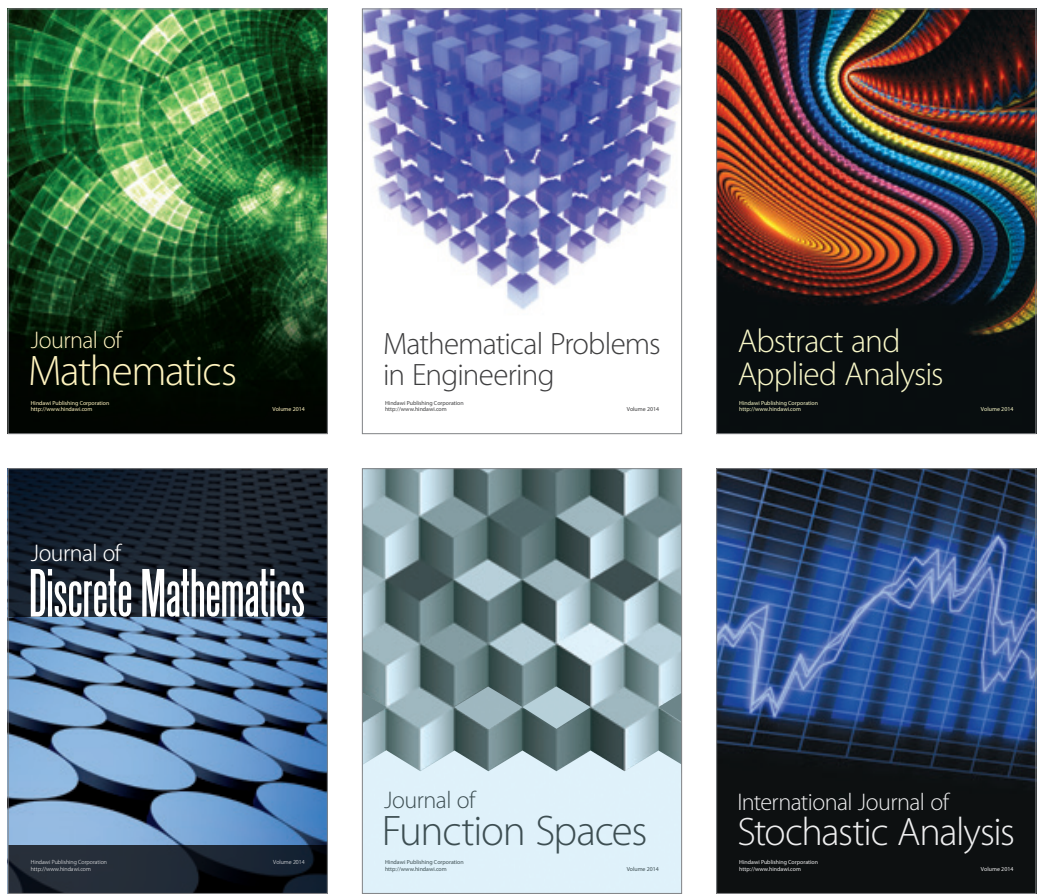

Journal of

Function Spaces

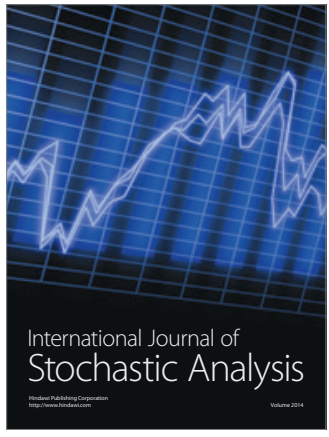

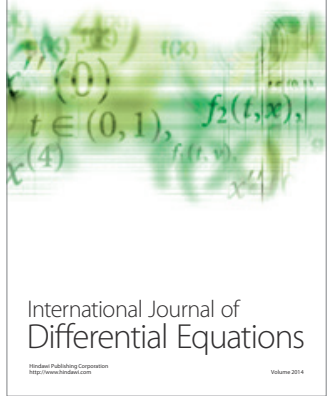
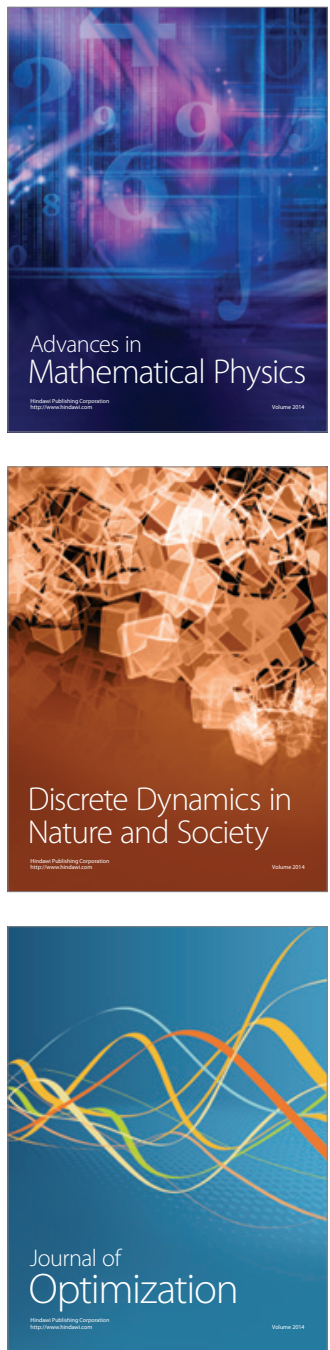